\title{
THE CULTURE OF WHOLE ORGANS
}

\section{Technique of the Culture of the Thyroid Gland}

BY ALEXIS CARREL, M.D.

(From the Laboratories of The Rockefeller Institute for Medical Research)

Plates 17 xo 19

(Received for publication, January 14, 1937)

The culture of whole organs (1) in the Lindbergh apparatus (2) has required the development of techniques that vary in some manner according to each organ. The purpose of this article is to describe the procedures used in the perfusion of the thyroid gland, the accidents that may happen during the operation, and the effects of the perfusion upon the gland.

\section{Technical}

The technique consists of several parts: preparation of the gaseous medium, preparation of the fluid medium, preparation of the Lindbergh perfusion pump, transplantation of the gland into the organ chamber, perfusion, and removal of the gland from the pump.

1. Preparation of the Gaseous Medium.-The gaseous medium is composed of nitrogen, oxygen, and carbon dioxide in various proportions. Generally, a mixture consisting of 80 per cent oxygen, 3 or 4 per cent carbon dioxide, and 16 or 17 per cent nitrogen is used. The mixture is made by introducing into a steel gas cylinder equipped with a pressure gauge the three gases under partial pressures of 80,3 , and 17 pounds, respectively. Thus, the cylinder contains the proper gas mixture under a pressure of 100 pounds. After a period of 24 hours, during which the gases are allowed to become thoroughly mixed, a gas pressure reducing valve is attached to the cylinder. When necessary, the composition of the mixture is ascertained by analysis. Before the experiment is started, the gas is led into the incubator and to the inner chamber of the oil flask (2). ${ }^{1}$

2. Preparation of the Fluid Medium.-The volume of the medium is approximately $200 \mathrm{cc}$. for a gland weighing $100 \mathrm{mg}$. It varies generally from $225 \mathrm{cc}$.

\footnotetext{
${ }^{1}$ Lindbergh (2), p. 425.
} 
to $250 \mathrm{cc}$. The medium consists of blood serum more or less diluted with Tyrode solution, or of artificial nutrient solutions containing only a small amount of blood serum. Phenol red is added at a concentration of $0.005 \mathrm{gm}$. per $100 \mathrm{cc}$. The fluid is sterilized by filtration. The filtering apparatus consists of an Erlenmeyer flask to which have been attached a lateral outlet for applying vacuum, and a curved fitting near the bottom of the flask for evacuating the fluid. Into the neck of the flask is introduced a Berkefeld filter mounted on a rubber stopper. The lateral outlet bears a rubber tube and a glass bulb filled with non-absorbent cotton. The lower outlet is connected by a short piece of rubber tubing, compressed by a clamp, with a cannula protected by a glass bell. The apparatus is prepared for sterilization by completely covering the bell with non-absorbent cotton enveloped in gauze. Then it is wrapped in paper and sterilized twice for 1 hour in an autoclave under a pressure of 17 to 18 pounds.

Before filtration, the $\mathrm{pH}$ of the fluid is brought to about 6.9 or 7.00 by bubbling through it a mixture of 92 per cent oxygen and 8 per cent carbon dioxide. The filtering apparatus is mounted on an appropriate stand. A funnel is connected by a short rubber tube with the Berkefeld filter. The lateral outlet is attached to the vacuum line through the bulb filter. The fluid is placed in the funnel and filtered into the flask. After filtration, the $\mathrm{pH}$ of the fluid is about 7.3 or 7.4. Careful examination shows whether there are any foreign particles floating in the field.

3. Preparation of the Lindbergh Perfusion Pump, and Introduction of the Fluid Medium into the Lower Reservoir.-The perfusion pump has been washed with chromic and sulfuric acid for 2 hours, and rinsed with running filtered water for 6 hours. Then it is dried by vacuum, the air being aspirated after double filtration, first, through a cotton bulb filter and second, through a Berkefeld filter. These operations should be carried out as described by Lindbergh (2). ${ }^{2}$ It is indispensable for the surface of the glass to be strictly clean, lest particles of dust form emboli in the small arteries and capillaries of the gland. Once thoroughly dried, the pump is prepared for sterilization by protecting all the openings with non-absorbent cotton and gauze plugs and pads. Then it is wrapped in paper. The rubber stoppers containing the glass tubes connected with the bulb filters $(2)^{\mathbf{3}}$ are prepared separately for sterilization in Petri dishes covered with gauze and cotton pads. The pump is placed in an autoclave, which is brought to a temperature of $160^{\circ} \mathrm{C}$. by introducing steam at a pressure of 75 pounds into the outer jacket. No steam is present in the sterilizing chamber. In case an autoclave of this type is not available, an electric oven can be used as a substitute. The sterilization lasts for 4 hours.

After the pump has cooled, the fluid medium is introduced into the reservoir chamber through the neck of the equalization chamber (2). ${ }^{4}$ The filtering appa-

\footnotetext{
${ }^{2}$ Lindbergh (2), p. 426, Operating Directions.

${ }^{3}$ Lindbergh (2), p. 415, Chart 2, Nos. 1, 2, 12, 22.

${ }^{4}$ Lindbergh (2), p. 415, Chart 2, No. 11.
} 
ratus is fixed to a stand in such a manner that the cannula and its bell are at the level of the neck of the equalization chamber of the pump. The gauze and cotton pads covering the neck of the pump and the bell of the filtering apparatus are removed simultaneously, and the cannula is introduced into the neck of the apparatus. This procedure renders infection practically impossible. Then the clamp is released and the fluid flows through the equalization chamber into the reservoir chamber. As the lateral outlet of the Erlenmeyer flask is protected by the bulb filter, there is no danger of contamination of the surface of the fluid by the air of the room. The necks of the equalization and reservoir chambers are now closed with rubber stoppers equipped with bulb filters, and sealed with waterproof cement. The opening of the organ chamber is left protected by cotton and gauze plugs and pads. When the fluid is subjected to quantitative analysis, it must be accurately measured. It is caused to flow from the filtering apparatus into a graduated tube. A funnel, protected by cellophane, is placed in the neck of the reservoir chamber $(2), 5$ and the fluid is poured into it, also under protection of cellophane.

4. Preparation of the Thyroid.-Cats and rabbits are generally used, less frequently, fowls. The technique is similar to that developed long ago for the transplantation in mass of organs (3). That is, the gland is removed, together with a segment of carotid artery in cats and rabbits, and of aorta in fowls, and with the surrounding connective tissue. The operative procedures require a higher degree of asepsis than an ordinary surgical operation, because an organ does not protect itself against infection in the perfusion pump as it does in the body. When the air of the room is not free from bacteria, antisepsis, as well as asepsis, must be used in the surgical procedures. During the handling of the blood vessels, special care is taken to prevent emboli due to the coagulation of the blood, to foreign bodies, or to air. As the thyroid artery of cats and rabbits is very small, it must be left undisturbed in the connective tissue and protected from antiseptics used during the operation. In sum, every detail of the technique must conform to the well known principles of vascular surgery.

(a) The skin of the neck of an etherized cat or rabbit is shaved, and sterilized with Dakin solution. Chloramine can be substituted for Dakin solution. Tincture of iodine is not used, because a large amount of iodine may go through the skin into the blood. The animal is bled to death from the femoral artery. The operating field is circumscribed with sterile black towels.

(b) At the time of death, or immediately after, a longitudinal incision from the chin to the sternum is made. The skin is dissected on each side and cut transversely in order that the flap can be everted. The trachea and muscles, and the edges of the operating field are scrupulously protected by pads squeezed out of antiseptic solution. In fowls, the left side of the thorax is opened along its whole length by an incision of the skin and muscles and section of the ribs.

(c) Incision of the muscles and exposure of the carotid artery and vagus nerve.

Lindbergh (2), p. 415, Chart 2, No. 18. 
The artery and the nerve are covered by a pad wet with Dakin solution. The aponeurosis is cut on the external side of the vagus nerve, which is not dissected from the artery. The vagus nerve is severed in the lower part of the neck and separated from the artery on a length of $1 \mathrm{~cm}$. A silk thread that has been soaked in Dakin solution is placed under the artery. This thread will later be used to tie the cannula in the artery. When a fowl is used, the heart, the aorta and its branches, and the thyroid are exposed by stretching the thoracic opening with a Gosset retractor. A silk thread is placed under the aorta.

(d) After ligature of the posterior collateral, which branches from the carotid at the level of the thyroid artery, the upper part of the vagus is severed. At a point located about $3 \mathrm{~cm}$. above the thyroid artery, a thread wet with Dakin solution is placed under the carotid and left provisionally untied. The connective tissue between the carotid artery, the vagus nerve, and the thyroid gland is undisturbed.

(e) Introduction of the cannula into the artery and perfusion of the gland. Glass cannulas of appropriate size, mounted on a piece of pure gum tubing, the total length of tubing and cannula being $5.5 \mathrm{~cm}$., have been sterilized in a small, round dish in Ringer solution. They lie full of fluid on the bottom of the dish. The solution has been filtered with extreme care, and is absolutely free from any floating particles. A Gentile syringe, the bulb separated from the glass chamber, has been sterilized in another jar. It is removed from the jar under the protection of a veil of sterile cellophane and filled with Ringer solution. This Ringer solution has been filtered through a Berkefeld filter and through a quartz sand filter, and must be completely free from floating particles. If any blood has remained in the carotid, it is pushed by pressure of the index finger toward the distal end of the artery. A small transverse incision is made with the scissors in the proximal part of the carotid. A cannula is taken with a mosquito forceps from the jar, the rubber tubing being clamped while the cannula is still under the fluid. In this manner, no air penetrates either into the cannula or into the rubber tubing, and the possibility of air embolus is removed. The cannula, protected against atmospheric dust by a gauze pad soaked in Dakin solution, is introduced into the artery, and fixed by tying the silk thread. One end of the thread is cut close to the knot. The other, at a distance of about $3 \mathrm{~cm}$. The same procedure is applied to the introduction of a cannula into the aorta of a fowl.

(f) Washing the blood out of the artery. The tip of the Gentile syringe is introduced into the rubber tubing of the cannula. The bulb is gently compressed in order to push the blood contained in the carotid artery and the small air bubbles that may have remained in the cannula into the distal part of the carotid, beyond the location of the future ligature. Then the artery is inspected along its entire length, in order to make sure that no foreign bodies, blood clots, or air bubbles are present. Afterwards, the thread lying on the distal part of the carotid artery is tied. By exercising more pressure on the bulb of the Gentile syringe, the gland is perfused with Ringer solution for a few seconds in such a 
manner as to expel all remaining blood. Then the syringe is removed while the bulb is being pressed, in order that the cannula and the rubber tubing may remain full of fluid. Care is taken afterwards not to make pressure on the rubber tubing in order to keep it full of fluid. In fowls, the blood of the aorta is

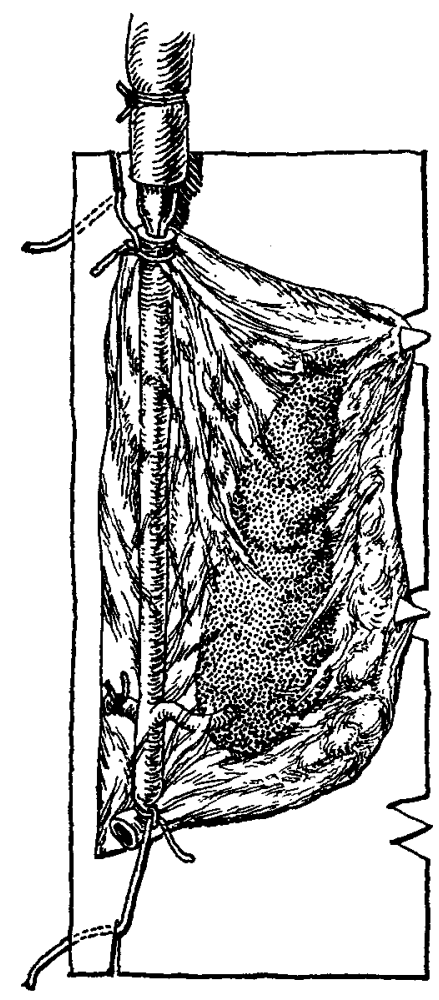

Texr-Fig. 1. Semischematic drawing. Mica holder with its upper and lower slits, and its three saw teeth. Carotid artery fixed on the holder by threads inserted in the slits. Gland suspended by attaching the surrounding connective tissue to saw teeth.

washed in the same manner. Then the distal ends of the arteries are ligated at a short distance from the edges of the thyroid.

(g) Section of the carotid and removal of the gland. The sternohyoid muscle is severed at the lower part of the neck, taken between the jaws of an artery forceps, and dissected out from the trachea and from the anterior surface of the thyroid gland to its upper insertion. Then it is cut and removed. The distal and proximal ends of the carotid artery are severed. The connective tissue is cut transversally from the carotid to the trachea. While the carotid and the 
gland are protected by a pad wet with Dakin solution, they are seized between the thumb and the index finger and pulled gently away from the trachea. The connective tissue is cut with scissors close to the trachea, the esophagus, and the muscles of the larynx. The gland is now removed in mass with the carotid and the intermediary connective tissue. The thyroid gland of the fowl is very easily removed with the large arteries on which it lies.

(h) Stretching the gland on a holder. The gland holder is made of a rectangular mica plate, 5.5 by $2.4 \mathrm{~cm}$. (Text-fig. 1). Close to one of the long sides of the plate, on each short side, there is a slit about $0.5 \mathrm{~cm}$. long. The other long side of the plate bears three sets of teeth, like saw teeth. The gland, protected by a pad, is stretched on the holder. The end of the thread that fixes the carotid to the cannula is inserted into the upper slit in the mica plate. The end of the thread of the distal ligature of the carotid is inserted into the lower slit (Text-fig. 1). In this manner, the carotid is maintained in a straight position without being stretched or twisted. Then the gland and its connective tissue are spread on the surface of the mica. Care is taken not to twist the connective tissue around the carotid segment or the thyroid artery. The thyroid artery is inspected from its beginning on the wall of the carotid artery to its division at the upper part of the gland. The outer part of the connective tissue is hung to the teeth of the bolder (Text-fig. 1). Then a large sheet of sterile cellophane is spread over the hands of the operator holding the mica plate, and the preparation is transported to the Lindbergh perfusion pump. The thyroid gland of the fowl is placed in the organ chamber without a holder being needed.

5. Setting the Gland in the Organ Chamber.-This consists of introducing the mica holder into the chamber, and of connecting the gland cannula with the feeding cannula.

(a) Introduction of the gland into the chamber. The operator stands in front of the culture chamber with the thyroid gland covered by the cellophane veil. An assistant simultaneously removes the pad and the plug protecting the opening of the chamber, and covers this opening with the edge of the cellophane veil. Under the protection of the cellophane, the mica plate is introduced into the chamber and allowed to slide down the tube as far as the platinum screen (2). In this manner, no infection from the air is possible.

(b) An assistant, by blowing through a rubber tube and bulb filter into the reservoir chamber, causes the fluid medium to flow through the feeding cannula into the organ chamber (2). ${ }^{2}$ The fuid must flow in such a manner that no foaming occurs either in the chamber itself, or in the feeding tube and cannula. When a sufficient amount of fluid has been introduced to wet the sand filter and fill the upper floating valve reservoir (2), ${ }^{2,7}$ the gland is mounted on the feeding cannula in the following manner. The upper part of the rubber tube is seized between the jaws of a curved forceps, and slipped on the feeding cannula.

${ }^{6}$ Lindbergh (2), p. 415, Chart 2, Nos. 4 and 5.

${ }^{7}$ Lindbergh (2), p. 415, Chart 2, Nos. 9 and 14. 
As little space as possible is left between the end of the feeding cannula and the beginning of the arterial cannula. Then, while the chamber is protected by the cellophane veil, the glass stopper, with its glass tubing and filter, is inserted into the opening.

6. Connecting the Pump with the Pulsating Apparatus.-(a) The three orifices of the pump are sealed carefully with waterproof cement. The apparatus is placed for a few minutes in the air current produced by a ventilator, in order to dry the cement. It is imperative for the sealing of the openings of the pump and of the points of insertion of the glass tubes into the rubber stoppers to be hermetic.

(b) The perfusion pump is placed in the incubator and connected with the pulsating gas pressure apparatus, in the way already described by Lindbergh (2). ${ }^{8}$ The pulse rate is adjusted, and also the maximum and minimum pressures, according to the nature of the experiment. The pulse rate has generally been 80 , the maximum pressure 160 , and the minimum pressure 110 .

7. Perfusion of the Gland.-The perfusion of the gland lasts about a week. During this period, the pulsation rate and the maximum and minimum pressures are observed every day and adjusted, if necessary. The modifications in the $\mathrm{pH}$ of the fluid are noted by comparison of the color of standard tubes with that of the feeding tube. Care is taken to prevent foaming and accumulation of the fluid in the chamber. There is need of very little adjustment of the apparatus during the period of perfusion. When the $\mathrm{pH}$ goes below 7 , the experiment is interrupted.

8. Removal of the Gland from the Chamber.-The gland is removed from the chamber either to be placed in another apparatus, or for the final results of the experiment to be studied. When an experiment is to be continued, the fresh pump is placed beside that containing the gland. After the cement has been incised with a knife, the rubber stopper is removed and the opening of the organ chamber carefully flamed with a Bunsen burner. Then both pumps are covered with a sheet of sterile cellophane. With a curved forceps, the gland is unhooked from the feeding cannula, removed from the chamber, and placed in the chamber of the second apparatus, as previously described. If the experiment is completed, the mica holder is removed, the gland dissected, weighed, and fixed in Zenker's formalin. The fluid is examined from the point of view of its physical changes, such as its clearness or opacity, and the presence or absence of floating particles. It is then removed from the pump and submitted to chemical analysis, which will be described in a subsequent article.

The extirpation of the thyroids of a cat or a rabbit, and their setting in two pumps takes about 40 minutes. The technique, in spite of its many details, is simple and can be learned easily in about 2 weeks.

${ }^{8}$ Lindbergh (2), p. 422. 


\section{Accidents}

The accidents that may happen to the thyroid during the course of the perfusion are infection, hemorrhage, emboli, and twisting or compression of the thyroid artery.

1. Infection generally is expressed by opacity of the fluid and change in its color. Nevertheless, in extremely rare cases there may be no visible modification of the fluid, the infection remaining confined to the gland, which becomes light yellow. The causes of infection have been bacteria or molds. This complication occurred almost exclusively during the first phase of the development of the technique. It disappeared when the pumps were sterilized at a higher temperature and for a longer time, and when the tissues were more adequately protected against contamination by the use during operations of gauze pads, gloves, thread, and instruments that had been antiseptically treated. The technique could be greatly simplified if the experiments were performed in a totally sterile room. However, in an atmosphere that is not free from bacteria, the above described technique has allowed a series of over 100 experiments to be made without an infection. Therefore, septic complications can be considered to be practically eliminated.

2. In order to be dangerous for the organ, a leakage has to be quito large. However, the posterior collateral of the cat's carotid artery, when not ligated, may cause a loss of fluid capable perhaps of bringing about a partial necrosis of the gland. Hemorrhages of smaller size are not dangerous. No ligatures of the collaterals of the thyroid artery, therefore, need to be made. These collaterals bleed into the connective tissue, which becomes more or less edematous. Such edema has no ill effect on the gland. Severe leakage is more apt to occur in the perfusion of the chicken's thyroid than in that of the cat's or rabbit's thyroid.

3. Emboli have more frequently brought about serious accident. They cause total or partial necrosis of the gland. Three types of emboli have been observed: foreign particles, blood clots, and gas bubbles. Sometimes the fluid used in the perfusion of the gland during the operation was found to contain floating particles. These particles may come from the Berkefeld filter, from the wall of the rubber tubing, from dust deposited by air inside of the pump or filtering apparatus, from cotton and gauze pads. All the rubber tubing used in the apparatus has to be of pure gum, in order that no foreign particles be set free into the fluid. The glassware is washed in filtered water, and dried in filtered air. Cellophane is interposed between the mouth of the jars containing Ringer solution and the pads, in order that the fluid may be protected against cotton or gauze filaments of the pads during sterilization. Finally, by filtering the fluid through quartz sand, and by allowing the particles that may come from the Berkefeld filter to collect on the bottom of the filtering flask, this type of embolus has been almost completely eliminated. Emboli may also come from a blood clot formed in the carotid artery if, after this artery is opened, the blood is not immediately washed 
out. The clots are generally large, and the necrosis of the gland is complete. This complication has been eliminated by strict adherence to the principles of blood vessel surgery. When the operation is performed on a dying or dead animal, the carotid artery is almost empty. If a small clot has formed in the carotid artery, it is forced by perfusion with the Gentile syringe into the distal part of the artery, beyond the location of the future ligature.

A large air embolus may also be a serious accident. The air can be introduced into the carotid from the organ cannula and its rubber tubing. This complication also takes place when air bubbles are present in the feeding cannula, or the feeding tube of the pump, or when the rubber tubing of the organ cannula is not completely filled with Ringer solution while it is being slipped over the feeding cannula. Simple procedures have been developed to prevent the occurrence of these accidents. By perfusing the carotid artery with the Gentile syringe before tying the ligature of the distal part of the artery, one causes the air bubbles that may remain in the lumen to be forced from the distal into the upper part of the vessel, beyond the distal thread. This thread must be tied only after inspection of the carotid artery has shown that it is free from blood clots or air bubbles. When the perfusion of the gland with the Gentile syringe is completed, the syringe is removed from the cannula in such a manner, as already mentioned, that the gland cannula and its rubber tubing contain no air bubbles. These various procedures have eliminated, in a large measure, the occurrence of partial or complete necrosis of the gland due to emboli.

4. Twisting or compression of the thyroid artery by connective tissue happens to cat and rabbit thyroids, and not to chicken thyroids. After the gland, its surrounding connective tissue, and the corresponding segment of carotid artery have been removed, the gland may become twisted around the carotid or the thyroid artery. This complication is prevented by spreading the preparation on the surface of the mica holder, taking pains to respect the normal relations of the vagus, the carotid, and the upper and lower poles of the gland. Each part of the preparation is maintained in its place by appropriate tension of the upper and lower arterial threads, and of the connective tissue hung on the mica teeth of the holder (Text-fig. 1). The thyroid artery must not be stretched, as in such a case the circulation of the gland is hampered. Another complication has been observed from connective tissue strands compressing the thyroid artery. This happens especially when the connective tissue has been made sticky by too persistent application of pads wet with Dakin solution. This arterial compression is prevented by examining the thyroid artery when it is spread on the holder, and cutting any strand of connective tissue that may compress it.

\section{Effects of Perfusion on the Gland}

As soon as the pump is connected with the second portion of the apparatus (2), ${ }^{9}$ and the maximum and minimum pressure are adjusted,

${ }^{\circ}$ Lindbergh (2), p. 427. 
the carotid artery begins to pulsate. Pulsation can also be detected in the thyroid artery. In a few minutes, the gland swells and becomes pink. There are little spurts of fluid from the small collaterals of the thyroid. Generally, these collaterals bleed into the connective tissue, which becomes more or less edematous. At the beginning of perfusion, the $\mathrm{pH}$ of the fluid in the feeding tube is about 7.5. It declines progressively in the course of a week to 7.1 or 7.0, and even lower, according to the metabolic activity of the gland and to the composition of the circulating fluid. The color of the gland becomes pinkishorange, orange, and yellow. It may be light yellow at the end of a week. Its size remains stationary, decreases, or increases, according to the composition of the medium. Often, these changes cannot be ascertained during perfusion, because the connective tissue is infiltrated by the circulating fluid flowing from small vessels.

The main effect of perfusion is to maintain the gland alive. The comparison of a gland cultivated for 6 days with the gland from the opposite side kept as control shows that there is practically no structural difference when the nutrient medium is diluted serum (Figs. 1 to 4). However, the cultivated glands show a tendency to hyperplasia and to vacuolization of the colloid. When the fluid contained some Witte's peptone, considerable increase in the size of the cells was observed (Figs. 5 and 6). The examination at higher power of the epithelium of perfused glands shows its excellent condition (Figs. 7 and 8). Marked proliferation of the epithelial cells lining the follicles was produced by a medium containing peptone (Figs. 9 and 10). Normal looking follicles were found after perfusions lasting 18 and 21 days. It is obvious that, although the circulating fluid contains neither red blood corpuscles nor hemoglobin, the thyroid does not show morphological signs of degeneration, but remains normal in appearance or undergoes enlargement and proliferation of the cells. Further evidence of the condition of the epithelial tissue after perfusion was given by the cultivation in flasks of small fragments of the glands. An exuberant culture of epithelial cells was obtained from a cat's gland through which diluted serum had circulated for 18 days (Fig. 11). In the same manner, although more rapidly, a very active migration of epithelial cells took place from a fragment of chicken gland, perfused for 3 days with a fluid containing Witte's peptone 
(Fig. 12). It is certain that thyroid glands perfused in the Lindbergh pump remain alive.

\section{CONCLUSIONS}

1. A technique has been developed for the transplantation of the whole thyroid into the Lindbergh pump, and its perfusion, without the occurrence of infection, emboli, and other complications.

2. The gland remains alive during the period of perfusion, which lasted from 3 to 21 days.

3. The technique is simple enough to be used as a routine procedure for physiological or pathological studies of the thyroid gland.

\section{BIBLIOGRAPHY}

1. Carrel, A., and Lindbergh, C. A., Science, 1935, 81, 621.

2. Lindbergh, C. A., J. Exp. Med., 1935, 62, 409.

3. Carrel, A., J. Exp. Med., 1908, 10, 98.

\section{EXPLANATION OF PLATES}

Plate 17

Frc. 1. Experiment 40. Left thyroid from cat KT kept as control; not cultivated, fixed in formol immediately after removal. $\times 230$.

FIG. 2. Experiment 40. Right thyroid from cat KT, perfused for 6 days with 40 per cent cat serum. Almost identical with the control. $\times 230$.

FIG. 3. Experiment 65. Left thyroid from cat MO kept as control; not cultivated, fixed in formol immediately after removal. $\times 230$.

FIG. 4. Experiment 65. Right thyroid from cat MO, perfused for 6 days with 40 per cent serum. Many vacuoles in colloid. Gland slightly hyperplastic. $\times 230$.

Fig. 5. Experiment 26. Left thyroid from cat KB kept as control; not cultivated, fixed immediately after removal. $\times 230$.

Fig. 6. Experiment 26. Right thyroid from cat KB, after perfusion for 6 days with medium containing Witte's peptone. Epithelial cells much larger than in control. Gland hyperplastic. $\times 230$.

\section{Piate 18}

Frg. 7. Experiment 274 L. Left thyroid from cat ST, perfused for 6 days with 50 per cent serum. The gland appears normal. $\times 480$.

Fig. 8. Experiment 305. Right thyroid from cat VA, perfused for 6 days with 40 per cent serum. Slightly hyperplastic gland. $\times 480$.

FIG. 9. Experiment 23. Right thyroid from adult chicken kept as control; not perfused, fixed in formol immediately after removal. $\times 230$. 
FIG. 10. Experiment 23. Left thyroid from same chicken perfused for 3 days with a medium containing Witte's peptone. Gland shows marked hyperplasia. $\times 230$.

\section{Plate 19}

Fig. 11. Culture in flask of a fragment of a cat's thyroid (Experiment 2), which had been perfused with 40 per cent serum for 18 days. Photograph shows the colony of epithelial cells developed after 6 days' cultivation. $\times 115$.

FIG. 12. Culture in flask of a fragment of an adult chicken thyroid (Experiment 8), which had been perfused for 8 days with a medium containing Witte's peptone. Photograph shows the colony of epithelial cells developed after 3 days' cultivation. $\times 115$. 

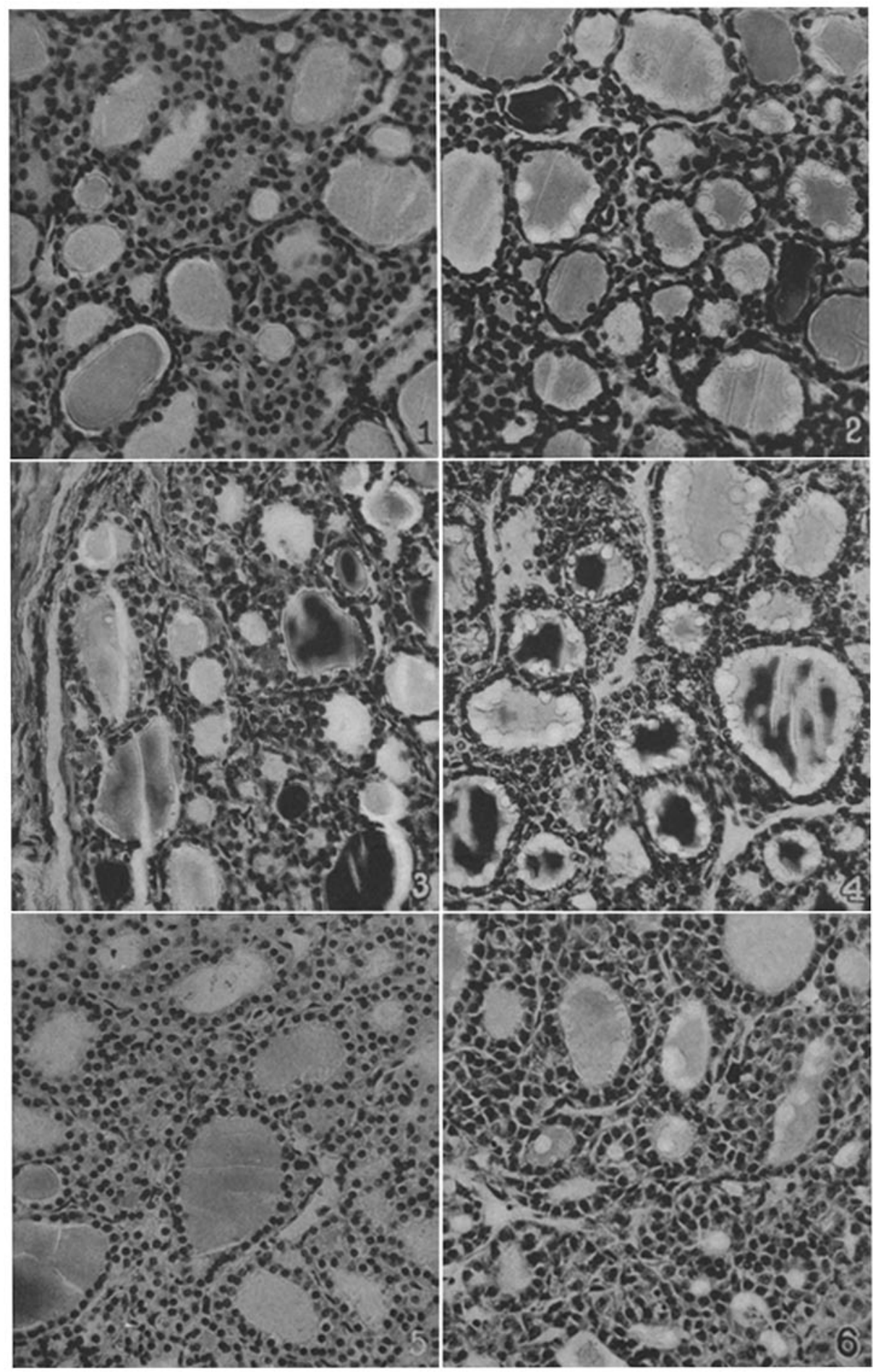

(Carrel: Culture of whole organs. I) 

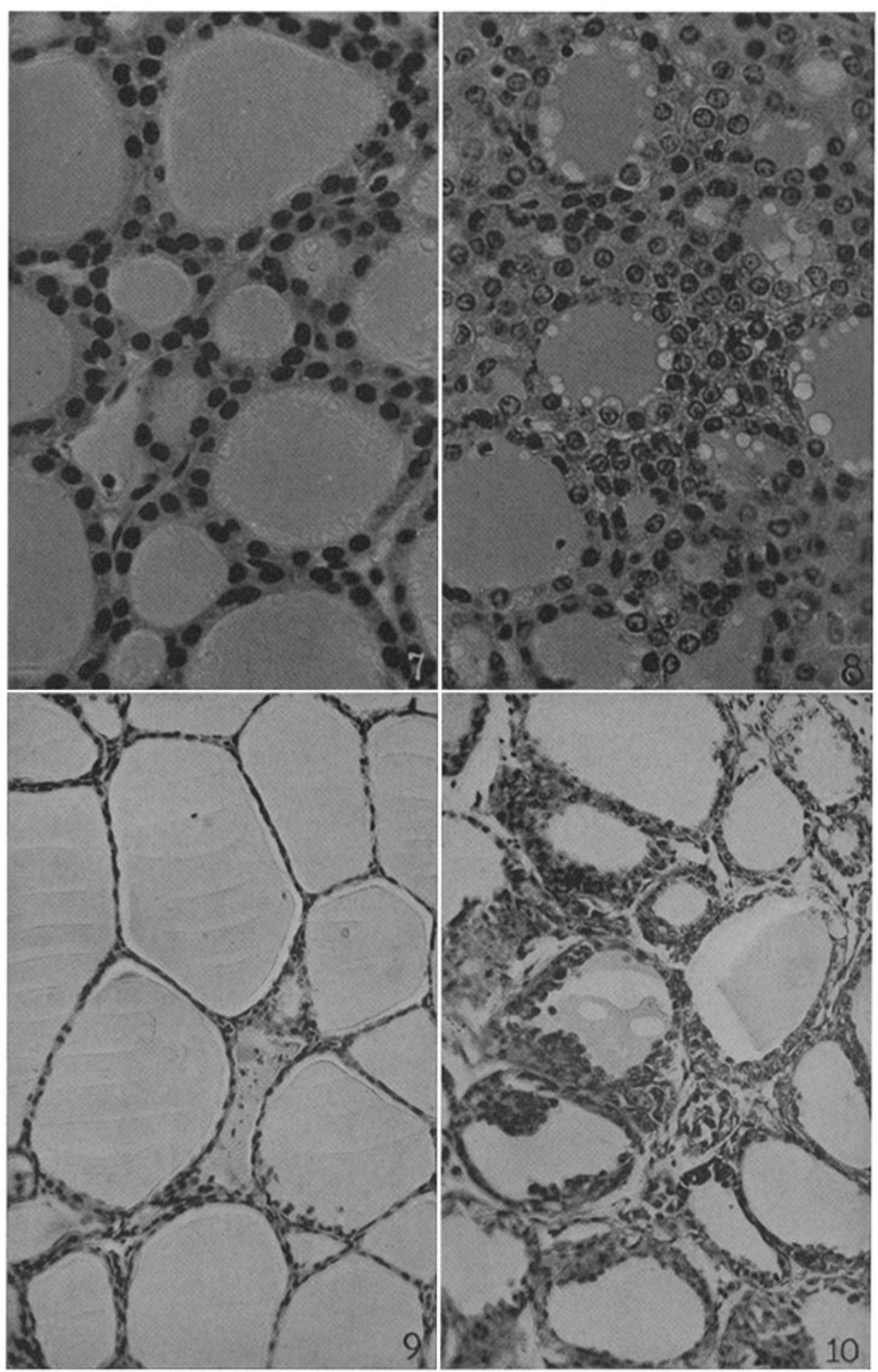

(Carrel: Culture of whole organs. I) 

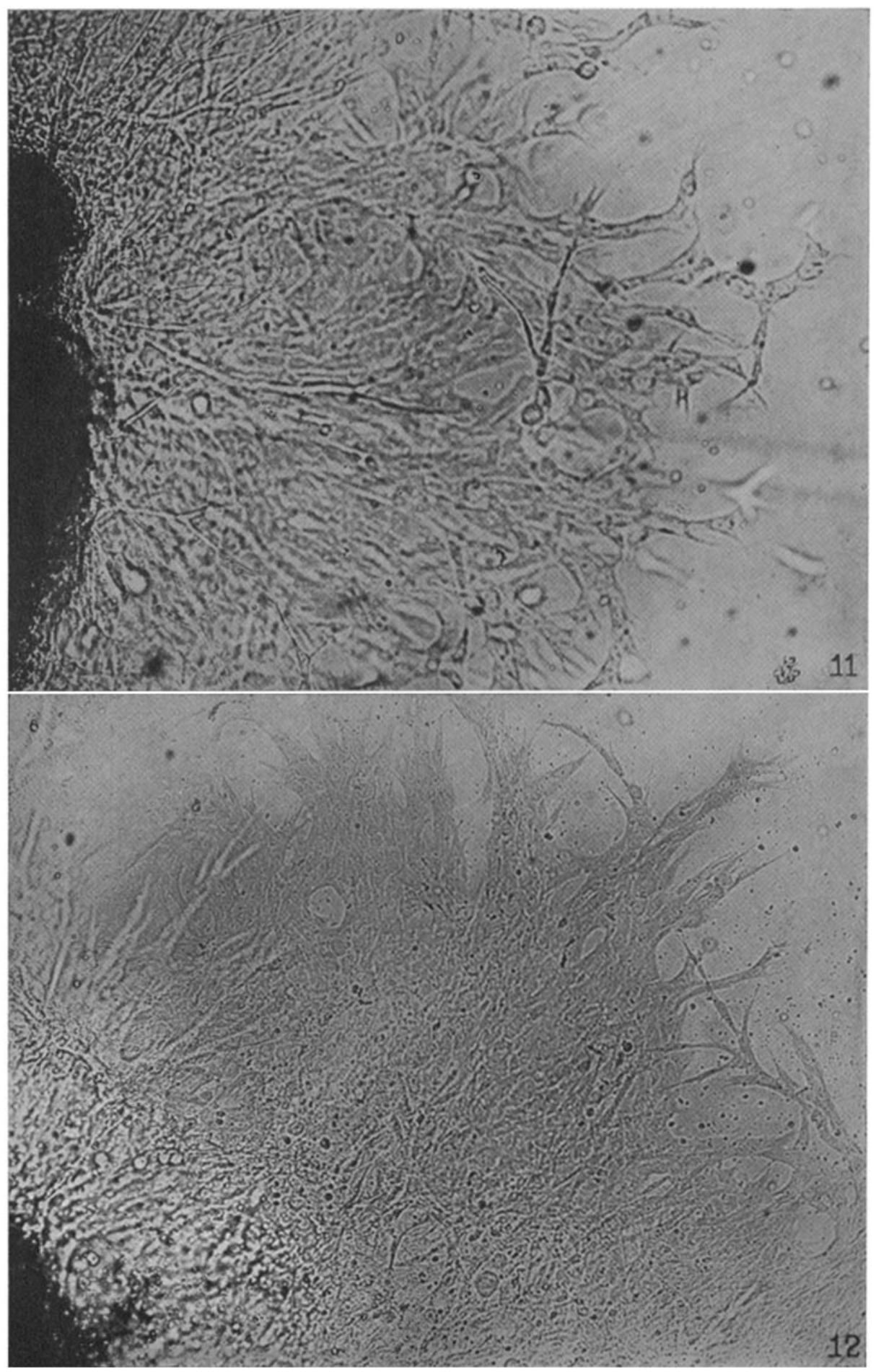

(Carrel: Culture of whole organs. I) 